\title{
LAMINAR MIXED CONVECTION FLOW FROM A VERTICAL SURFACE WITH INDUCED MAGNETIC FIELD AND CONVECTIVE BOUNDARY
}

\author{
K. CHAUDHARY and A. SHARMA \\ Department of Mathematics, University of Rajasthan \\ Jaipur - 302004, INDIA \\ A.K. JHA ${ }^{*}$ \\ Department of Mathematics, J.N. College Madhubani Bihar \\ 847211 INDIA \\ E-mail: itsabhay@rediffmail.com
}

\begin{abstract}
The objective of this investigation is to study the influence of thermal radiation and radiation absorption parameter on a mixed convection flow over a continuously moving porous vertical plate under the action of transverse applied magnetic field taking into account the induced magnetic field with convective boundary. Under certain assumptions, the solutions for the velocity field, temperature distribution and induced magnetic field are obtained. The influences of various parameters on the velocity, temperature fields and on induced magnetic fields are studied graphically. It is also found that the dimensionless Prandtl number, Grashof number, Schmidt number and magnetic parameter have an appreciable influence on the independent variables.
\end{abstract}

Key words: thermal radiation, radiation absorption, convective boundary, induced magnetic field.

\section{Introduction}

The convective heat and mass transfer processes with phase change include evaporation of a liquid at the interface between a gas and a liquid or sublimation at a gas-solid interface. They can be described using the method for convective heat and mass transfer. In many engineering applications, heat and mass transfer processes in fluids condensing or boiling at a solid surface play a decisive role. Magneto-hydrodynamics is the branch of continuum mechanics, which deals with the flow of electrically conducting fluids in electric and magnetic fields. The study of forced and free convection flow and heat transfer for electrically conducting fluids past a semi infinite porous plate under the influence of a magnetic field has attracted the interest of many investigators. MHD free convection flows have great significance for the application in the field of satellite and planetary magnetospheres, aeronautics and chemical engineering.

This subject has been investigated by many researchers. Soundalgekar [1,2] studied the MHD free convection flow past a vertical porous plate. Kafousias [3] investigated the MHD free convection effects on Stokes problem for an incompressible viscous fluid flowing past an infinite vertical limiting surface. Raptis and Kafoussias [4] studied magnetohydrodynamic free convection flow and mass transfer through a porous medium bounded by an infinite vertical porous plate with constant suction. Mass transfer effects on an unsteady free convective flow past an infinite vertical porous plate with variable suction was analyzed by Ramana Kumari and Bhaskara Reddy [5]. Elbashbeshy [6] studied the mixed convection along a vertical plate embedded in a non-Darcian porous medium with suction and injection. Makinde [7] investigated a free convection flow with thermal radiation and mass transfer past a moving vertical porous plate. A transient magnetohydrodynamic free convective heat and mass transfer flow with thermophoresis past a radiate inclined permeable plate in the presence of variable chemical reaction and temperature dependent viscosity

\footnotetext{
* To whom correspondence should be addressed
} 
was studied by Alam et al. [8]. Ahmed Sahin [9] investigated a free and forced convective MHD oscillatory flow over an infinite porous surface in an oscillatory free stream. Gireesh Kumar and Satyanarayana [10] analyzed the mass transfer effect on MHD unsteady free convective Walters's memory flow with constant suction and heat sink.

The above studies have generally assumed a very small magnetic field Reynolds numbers, allowing magnetic induction effect to be neglected. The influence of the induced magnetic field on various problems has been examined. Megakhed [11] examined the effect of the induced magnetic field and heat transfer on a non stationary magnetohydrodynamic flow around a porous plate. Chaudhary and Bhupendra [12] examined the combined heat and mass transfer by laminar mixed convection flow from a vertical surface with the induced magnetic field. Alom et al. $[13,14]$ investigated similarity solutions for a hydromagnetic free convective heat and mass transfer flow along a semi-infinite permeable inclined flat plate with heat generation and thermophoresis. Bég et al. $[15,16]$ analyzed a non- similar, laminar, steady, electricallyconducting forced convection liquid metal boundary layer flow with the induced magnetic field. Ahmed [17, $18]$ studied the induced radiating fluid flow past a porous vertical plate.

In recent investigations, several researchers have shown interest in obtaining solutions of boundary layer flows over flat surfaces with convective boundary conditions. Batler [19] investigated radiation effects for the Blassius and Sakiadis flows with a convective surface boundary condition. Makinde and Aziz [20] studied MHD mixed convection from a vertical plate embedded in a porous medium with a convective boundary condition. Heat transfer of a generalized stretching/shrinking wall problem with convective boundary conditions was examined by Yao and Zhong [21]. Sivraj and Rushi kumar [22] studied a chemically reacting dusty viscoelastic fluid flow in an irregular channel with convective boundary.

Radiative convective flows are encountered in countless industrial and environmental processes, e.g. nuclear power plants, gas turbines heating and cooling chambers, fossil fuel combustion and various propulsion devices for air crafts, missiles and space vehicles. Hossain and Takhar [23] studied the radiation effect on mixed convection along a vertical plate with uniform surface temperature. Raptis and Massalas [24] analyzed a magnetohydrodynamic flow past a plate in the presence of radiation. Raptis and Perdikis [25] studied thermal radiation of an optically thin gray gas. Effects of radiation in an optically thin gray gas flowing past a vertical infinite plate in the presence of a magnetic field were analyzed by Raptis et al. [26, 27]. Ahmed and Tridip [28] investigated thermal radiation and magnetohydrodynamic effects on heat and mass transfer of a chemically reacting fluid with periodic suction. Prasad et al. [29] studied thermal radiation effects on magnetohydrodynamic free convection heat and mass transfer from a sphere in a variable porosity regime.

In recent years, the convective flow with simultaneous heat and mass transfer under the influence of a magnetic field and chemical reaction, has attracted considerable attention of researchers. These processes are widely used in many industrial applications such as food processing, manufacturing of ceramics polymer production, drying evaporation at the surface of a water body and electric power industry. Takhar et al. [30] investigated the flow and mass transfer on a stretching sheet with a magnetic field and chemically reactive species. Muthucumarswamy and Ganesan [31,32] studied- the first order chemical reaction on flow past an impulsively started vertical plate with uniform heat and mass flux. Chemical reaction, heat and mass transfer on an MHD flow over a vertical stretching surface with heat source and thermal stratification effects were studied by Kandasamy et al. [33]. Ibrahim et al. [34] investigated the effect of chemical reaction and radiation absorption on the unsteady MHD free convection flow past a semi infinite vertical permeable moving plate with heat source and suction. Prakash et al. [35] analyzed the influence of chemical reaction on an unsteady MHD mixed convective flow over a moving vertical porous plate. Combined effects of Joule heating and chemical reaction on unsteady magnetohydrodynamic mixed convection of a viscous dissipating fluid over a vertical plate in porous media with thermal radiation were studied by Pal and Talukdar [36].

The aim of this paper is to investigate the thermal radiation effect, radiation absorption effect on a combined heat and mass transfer of a steady laminar mixed free-forced convective flow of a viscous incompressible electrically conducting fluid above a semi-infinite vertical porous surface under the influence of an induced magnetic field and convective boundary. The perturbation technique is used to solve the problem. The results for the Nusselt number, Sharewood number and skin friction are obtained and discussed graphically. 


\section{Formulation of the problem}

The steady mixed convection heat and mass transfer flow of a viscous, incompressible, electrically conducting fluid past a continuously moving infinite vertical porous plate under the influence of a transversely applied magnetic field is considered. Following the Cartesian coordinate system, the flow is assumed to be in the $x$ - direction, which is taken along the vertical plate in the upward direction, whereas the $y$ - direction is normal to the plate. The magnetic field is of the form $H=\left(H_{x}, H_{y}, 0\right)$. The equation of conservation of the electric charge is $\nabla . J=0$, where $J=\left(J_{x}, J_{y}, J_{z}\right)$. Since the direction of propagation of the electric charge is along the $y$-axis and the plate is electrically non-conducting, $J_{y}=0$ everywhere within the flow. It is also assumed that the Joule heating effect is small enough and divergence equation for the magnetic field $\nabla . H=0$ is of the form $H_{y}=H_{0}$. The schematic diagram of the flow configuration and the coordinate system of the problem are shown in the figure below.

Further, as the plate is of infinite length, all physical variables depend on $y$ only. and therefore the equation of continuity is given by

$$
d v / d y=0 .
$$

$\Rightarrow v=-V_{0}$ where $V_{0}$ is the constant velocity of suction normal to the plate and the negative sign indicates that the suction velocity is directed towards the plate surface.

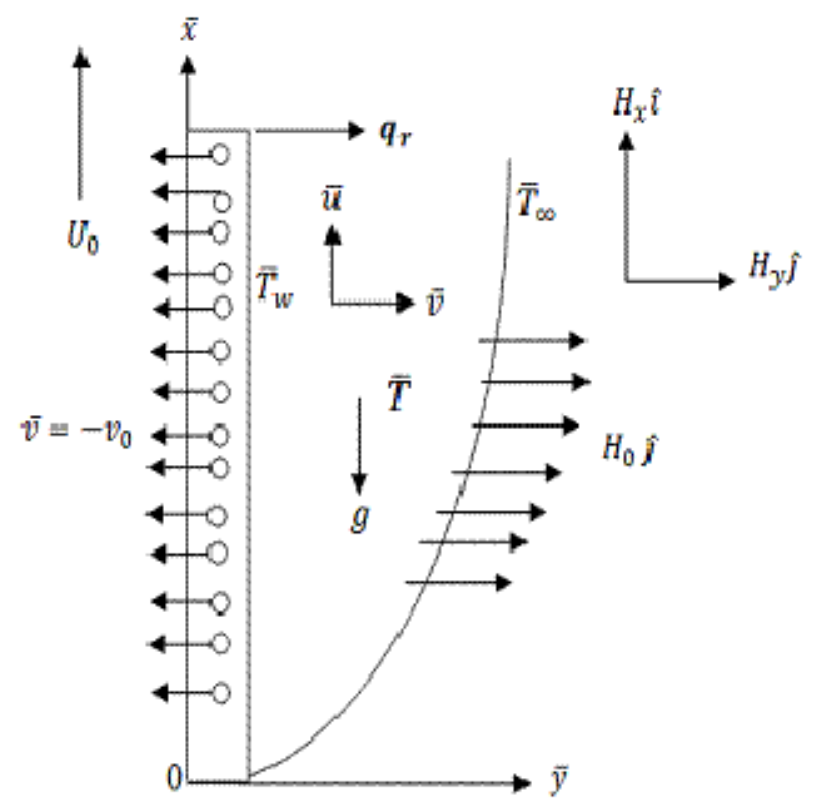

Fig.1. Physical model and coordinate system.

In accordance with the above assumptions and under usual Boussinesq's approximation, the basic equations related to the problem and generalized Ohm's law can be put in the following form:

Momentum equation

$$
-V_{0} d u / d y=g \beta\left(T-T_{\infty}\right)+g \beta^{*}\left(C-C_{\infty}\right)+v d^{2} u / d y^{2}+\frac{\mu_{e} H_{0}}{\rho} \frac{d H_{x}}{d y} .
$$


The induced magnetic field

$$
-V_{0} d H_{x} / d y=H_{0} d u / d y+\frac{1}{\sigma \mu_{e}} \frac{d^{2} H_{x}}{d y^{2}} .
$$

Energy equation

$$
-V_{0} \frac{d T}{d y}=\frac{k}{\rho C_{p}} \frac{d^{2} T}{d y^{2}}+\frac{\vartheta}{C_{p}}\left(\frac{d u}{d y}\right)^{2}+\frac{1}{\sigma \rho C_{p}}\left(\frac{d H_{x}}{d y}\right)^{2}-\frac{1}{\rho C_{P}} \frac{d q}{d y}+\frac{Q_{C}}{\rho C_{P}}\left(C_{W}-C_{\infty}\right) .
$$

Concentration equation

$$
-V_{0} \frac{d C}{d y}=D \frac{d^{2} C}{d y^{2}}-\lambda\left(C_{W}-C_{\infty}\right) .
$$
follows

The relevant boundary conditions on the vertical surface and in the uniform stream are defined as

$$
\begin{aligned}
& u=U_{0}, \quad-k \frac{d T}{d y}=-h_{f}\left(T_{\infty}-T\right), \quad \frac{d C}{d y}=-\frac{m}{D}, \quad H_{x}=H_{w} \quad \text { at } y=0, \\
& u=0, \quad T=T_{\infty}, \quad C=C_{\infty}, \quad H_{x}=0 \quad \text { when } \quad y \rightarrow \infty .
\end{aligned}
$$

The radiative heat flux is given by

$$
\frac{\partial q}{\partial y}=4\left(T_{\infty}-T\right) I_{l}^{\prime}, \quad I^{\prime}=\int_{0}^{\infty} K_{\lambda_{l} W} \frac{\partial e_{b \lambda_{l}}}{\partial T} d \lambda_{l}
$$

where $g$ is the acceleration due to gravity, $\beta$ is the coefficient of thermal expansion, $T$ denotes the fluid temperature, $C$ is the concentration of species, $T_{\infty}$ and $C_{\infty}$ are the temperature and species concentration of the uniform flow, $\beta^{*}$ is the concentration expansion coefficient, $\vartheta$ is the Newtonian kinematic viscosity of the fluid, $\mu_{e}$ is the magnetic permeability, $H_{0}$ is the applied constant magnetic field, $H_{x}$ is the induced magnetic field, $\rho$ is the density of fluid, $\sigma$ is the electrical conductivity, $k$ is the thermal conductivity, $D$ is the chemical molecular diffusivity, $C_{p}$ is the specific heat capacity of the fluid at constant temperature, $C_{S}$ is the concentration susceptibility, $k_{T}$ is the thermal diffusion ratio, $U_{0}$ is the uniform velocity, $Q$ is the constant heat flux per unit area, $\mathrm{m}$ is the constant mass flux per unit area and $H_{w}$ is the induced magnetic field at the wall, respectively. In order to simplify a numerical solution, we introduce the following transformations, viz 


$$
\begin{aligned}
& u^{*}=\frac{u}{V_{0}}, \quad y^{*}=\frac{V_{0} y}{\vartheta}, \quad U^{*}=\frac{U_{0}}{V_{0}}, \quad k^{*}=\frac{V_{0}^{2} k}{\vartheta^{2}}, \\
& C^{*}=\frac{D V_{0}\left(C-C_{\infty}\right)}{\vartheta m}, \quad \theta^{*}=\frac{k V_{0}\left(T-T_{\infty}\right)}{\vartheta Q}, \quad H^{*}=\sqrt{\frac{\mu_{e}}{\rho}} \frac{H_{x}}{V_{0}}
\end{aligned}
$$

and define the following dimensionless parameters

$$
\begin{aligned}
\operatorname{Pr} & =\frac{\rho \vartheta C_{p}}{k}(\text { P randtl number }), \\
\mathrm{Gr} & =\frac{\vartheta^{2} g \beta Q}{k V_{0}^{4}}(\text { Grashoff number }), \\
\mathrm{Gm} & =\frac{\vartheta g \beta^{*}\left(C_{w}-C_{\infty}\right)}{V_{0}^{3}}(\text { modified Grashoff number }), \\
\mathrm{Ec} & =\frac{k V_{0}^{3}}{\vartheta Q C_{p}}(\text { Eckert number }), \\
\alpha_{C} & =\frac{Q_{C}\left(C_{W}-C_{\infty}\right) V_{0}^{2}}{k\left(T_{W}-T_{\infty}\right)} \text { radiationa absorption parameter, } \\
\mathrm{Dr} & =\frac{\rho m k_{T}}{Q C_{s}}(\text { diffusion-thermal or Dufour number }), \\
\mathrm{Sc} & =\frac{\vartheta}{D}(\mathrm{Schmidt} \text { number }), \\
P_{m} & =\sigma \vartheta \mu_{e}(\text { magnetic diffusivity }), \\
M & =\frac{H_{0}}{V_{0}} \sqrt{\frac{\mu_{e}}{\rho}}(\text { magnetic parameter }), \\
V_{0}^{2} & \text { radiation parameter } \\
&
\end{aligned}
$$

where $C_{w}$ is the concentration on the plate wall.

Therefore, substituting Eqs (2.7) in Eqs (2.2)-(2.5) with boundary conditions (2.6) and introducing the above non- dimensional quantities and ignoring the asterisk $(*)$, we obtain 


$$
\begin{aligned}
& \frac{d^{2} u}{d y^{2}}+M \frac{d H}{d y}+\frac{d u}{d y}=-\mathrm{Gr} \theta-\mathrm{Gm} C, \\
& \frac{1}{P_{m}} \frac{d^{2} H}{d y^{2}}+M \frac{d u}{d y}+\frac{d H}{d y}=0, \\
& \frac{d^{2} \theta}{d y^{2}}+\operatorname{Pr} \frac{d \theta}{d y}-F \theta=-\operatorname{Pr} \operatorname{Ec}\left[\left(\frac{d u}{d y}\right)^{2}+\frac{1}{P_{m}}\left(\frac{d H}{d y}\right)^{2}\right]+\alpha_{c} C, \\
& \frac{1}{\mathrm{Sc}} \frac{d^{2} C}{d y^{2}}+\frac{d C}{d y}-C^{*} \lambda=0 .
\end{aligned}
$$

With transformed boundary conditions

$$
\begin{aligned}
& y=0: u=U, \quad \frac{d \theta}{d y}=B_{i}(\theta-1), \quad \frac{d C}{d y}=-1, \quad H=h, \\
& y \rightarrow \infty: u=0, \quad \theta=0, \quad C=0, \quad H=0
\end{aligned}
$$

where $\theta$ is the dimensionless temperature and $U$ is the velocity of the moving plate and $h=\frac{H_{w} M}{H_{0}}$.

\section{Solution of the problem}

To obtain a complete solution of the coupled nonlinear system of Eqs (2.8) -(2.10) under boundary conditions (2.12), we introduce the perturbation approximation. Since the dependent variables $u, H$ and $\theta$ mostly dependent on $y$ only and the fluid is purely incompressible one, we expand the dependent variables in powers of the Eckert number Ec which is small enough such that the terms in $E_{c}^{2}$ and their higher order powers $E_{c}^{2}$ can be neglected. Thus, we assume

$$
\begin{aligned}
& u(y)=u_{l}(y)+\operatorname{Ec} u_{2}(y)+O\left(E_{c}^{2}\right)+\ldots \ldots \\
& H(y)=H_{l}(y)+\operatorname{Ec} H_{2}(y)+O\left(E_{c}^{2}\right)+\ldots \\
& \theta(y)=\theta_{l}(y)+\operatorname{Ec} \theta_{2}(y)+O\left(E_{c}^{2}\right)+\ldots \ldots
\end{aligned}
$$

Now substituting (3.1) into Eqs (2.8) - (2.10), neglecting the terms of $E_{c}^{2}$ and higher order powers and equating the coefficient of like powers of Ec, we obtain the equations of first and second order approximations as given below:

Coefficient of $(\mathrm{Ec})^{0}$ 


$$
\begin{aligned}
& \frac{d^{2} u_{1}}{d y^{2}}+M \frac{d H_{1}}{d y}+\frac{d u_{1}}{d y}=-\mathrm{Gr} \theta_{1}-\frac{\mathrm{Gm}}{m_{1}} e^{-m_{1} y}, \\
& \frac{d^{2} H_{1}}{d y^{2}}+P_{m} \frac{d H_{1}}{d y}+M P_{m} \frac{d u_{1}}{d y}=0, \\
& \frac{d^{2} \theta_{1}}{d y^{2}}+\operatorname{Pr} \frac{d \theta_{1}}{d y}-F \theta=\alpha_{C} C,
\end{aligned}
$$

with boundary conditions

$$
\begin{aligned}
& y=0: u_{1}=U_{0}, \quad \frac{d \theta_{1}}{d y}=B_{i}(\theta-1), \quad H_{1}=h, \\
& y \rightarrow \infty: u_{1}=0, \quad \theta_{1}=0, \quad H_{1}=0 .
\end{aligned}
$$

Coefficient of $(\mathrm{Ec})^{1}$

$$
\begin{aligned}
& \frac{d^{2} u_{2}}{d y^{2}}+M \frac{d H_{2}}{d y}+\frac{d u_{2}}{d y}=-\operatorname{Gr} \theta_{2}-\frac{1}{m_{1}} e^{-m_{1} y}, \\
& \frac{d^{2} H_{2}}{d y^{2}}+P_{m} \frac{d H_{2}}{d y}+M P_{m} \frac{d u_{2}}{d y}=0, \\
& \frac{d^{2} \theta_{1}}{d y^{2}}+\mathrm{P}_{\mathrm{r}} \frac{d \theta_{1}}{d y}-F \theta=-\operatorname{Pr}\left(u_{1}^{\prime}\right)^{2}-\frac{\operatorname{Pr}}{P m}\left(H_{1}^{\prime}\right)^{2},
\end{aligned}
$$

with boundary conditions

$$
\begin{aligned}
& y=0: u_{2}=0, \quad \frac{d \theta_{2}}{d y}=B_{i} \theta_{2}, \quad H_{1}=0, \\
& y \rightarrow \infty: u_{2}=0, \quad \theta_{2}=0, \quad H_{2}=0 .
\end{aligned}
$$

Thus, solving the above Eqs (3.2) - (3.4) under boundary conditions (3.5), we obtain

$$
\begin{aligned}
& \theta_{1}=A_{1} e^{-m_{2} y}+A_{2} e^{-m_{1} y}, \quad H_{1}=A_{6} e^{-m_{3} y}+A_{3} e^{-m_{1} y}+A_{4} e^{-m_{2} y}, \\
& u_{1}=A_{10} e^{-y}+A_{9} e^{-m_{3} y}+A_{8} e^{-m_{1} y}+A_{7} e^{-m_{2} y} .
\end{aligned}
$$

Similarly, solving Eqs (3.6) - (3.8) with boundary conditions (3.9), we get 


$$
\begin{aligned}
& \theta_{2}=A_{21} e^{-m_{2} y}+A_{11} e^{-2 m_{2} y}+A_{12} e^{-2 m_{1} y}+A_{13} e^{-2 m_{3} y}+A_{14} e^{-\left(m_{1}+m_{2}\right) y}+A_{15} e^{-\left(m_{3}+m_{2}\right) y}+ \\
& +A_{16} e^{-\left(m_{1}+m_{3}\right) y}+A_{17} e^{-2 y}+A_{18} e^{-\left(1+m_{2}\right) y}+A_{19} e^{-\left(1+m_{1}\right) y}+A_{20} e^{-\left(1+m_{3}\right) y} \\
& H_{2}=A_{33} e^{-m_{3} y}+A_{22} e^{-m_{2} y}+A_{23} e^{-2 m_{2} y}+A_{24} e^{-2 m_{1} y}+A_{25} e^{-2 m_{3} y}+A_{26} e^{-\left(m_{1}+m_{2}\right) y}+ \\
& +A_{27} e^{-\left(m_{3}+m_{2}\right) y}+A_{28} e^{-\left(m_{1}+m_{3}\right) y}+A_{29} e^{-2 y}+A_{30} e^{-\left(1+m_{2}\right) y}+A_{31} e^{-\left(1+m_{1}\right) y}+A_{32} e^{-\left(1+m_{3}\right) y} \\
& u_{2}=A_{45} e^{-y}+A_{33 a} e^{-m_{2} y}+A_{34} e^{-2 m_{2} y}+A_{35} e^{-2 m_{1} y}+A_{36} e^{-2 m_{3} y}+ \\
& +A_{37} e^{-\left(m_{1}+m_{2}\right) y}+A_{38} e^{-\left(m_{3}+m_{2}\right) y}+A_{39} e^{-\left(m_{1}+m_{3}\right) y}+A_{40} e^{-y}+A_{41} e^{-\left(1+m_{2}\right) y}
\end{aligned}
$$

The current density $(J)$ is given by

$$
J=-\left(\frac{\partial H}{\partial y}\right)
$$

The shearing stress at the plate is given by $\left(\frac{\partial u}{\partial y}\right)_{y=0}=\left(\frac{\partial u_{1}}{\partial y}\right)_{y=0}+\left(\frac{\partial u_{2}}{\partial y}\right)_{y=0}$.

Heat flux $(\mathrm{Nu})=-\left(\frac{\partial T}{\partial y}\right)_{y=0}$

\section{Result and discussion}

The formulation of the effect of the thermal radiation, radiation absorption parameter, convective and the dimensionless thermo physical parameters boundary conditions on a free convection heat and mass transfer flow of a viscous incompressible electrically conducting fluid along a semi-infinite vertical plate, under the action of a transverse applied magnetic field taking into account the induced magnetic field has been given in the preceding sections. This enables us to carry out the numerical calculations for distributions of the velocity field, temperature field, induced magnetic field, rate of heat transfer, skin friction and electric current density at the plate. In the present study, the physical parameters are taken as (Prandtl number) $\mathrm{Pr}=$.71, Gr (Grashof number)=2, (magnetic diffusivity) $P m=1$, (Eckert number) Ec=.001, (magnetic field) $M=1$, (radiation absorption parameter) $\alpha_{c}=1$, (chemical reaction parameter) $\lambda=4, U_{0}=1$, (radiation parameter) $F=1$, (Schmidt number) $\mathrm{Sc}=.33$, (modified Grashof number) $\mathrm{Gm}=3$, (Biot number) $\mathrm{Bi}=.5$.

Figures 1 and 2 depict the velocity profiles and induced magnetic profiles for $\operatorname{Pr}=.71$ (air at $20^{\circ} \mathrm{C}$ ) for different values of the Biot number $(\mathrm{Bi})$, chemical reaction parameter $(\lambda)$ and heat absorption parameter $\left(\alpha_{c}\right)$. We can see that the velocity profile notably decreases for higher values of the Biot number, it is also noticed that an increase in the chemical reaction parameter and radiation absorption parameter leads to the increase in velocity. A distinct velocity escalation occurs near the wall after which profiles decay smoothly to the stationary value in free stream. Chemical reaction therefore boosts momentum transfer i.e., accelerates the flow. Further, Fig. 2 shows that an increase in the Biot number parameter leads to a decrease in the induced magnetic field whereas an increase in the radiation absorption parameter and chemical reaction parameter leads to an increase in the induced magnetic field. 




Fig.1. Effect of $\mathrm{Bi}, \lambda$ and $\alpha_{c}$ on velocity.

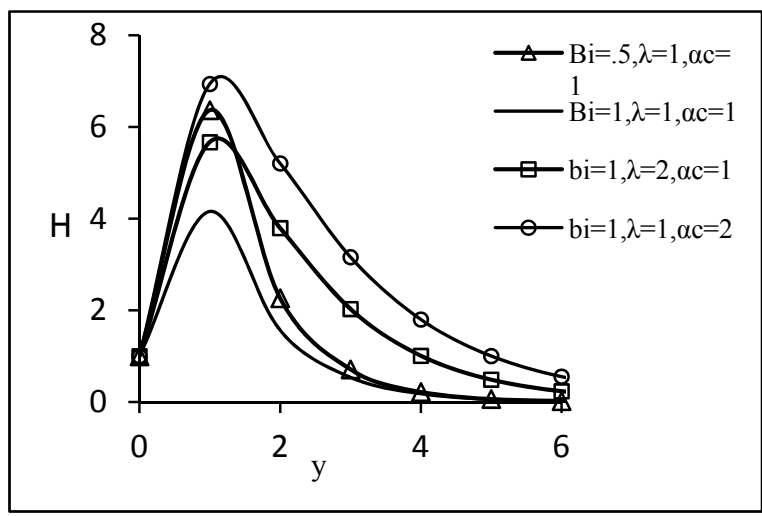

Fig.2. Effect of $\mathrm{Bi}, \lambda$ and $\alpha_{c}$ on induced magnetic field.

Figures 3 and 4 are plotted to show the influence of the Schmidt number (Sc) on velocity and the induced magnetic field. It can be seen that both profiles show a similar variation that is larger for helium $\left(\mathrm{Sc}=.30\right.$ at temperature $25^{\circ}$ and 1 atmosphere pressure) than ammonia $(\mathrm{Sc}=.78)$, at temperature $25^{\circ} \mathrm{C}$ and 1 atmosphere pressure). As might be expected, the velocity profiles increase gradually near the plate, become the highest in the vicinity of the plate, and then decrease slowly away from the plate. The velocity profile and induced magnetic field profile in Figs 5 and 6 are drawn for cooling Newtonian fluid $(\mathrm{Gr}>0)$. It shows that an increase in the Grashof number increases the velocity which is taking place through the application of a pressure gradient but a reverse effect is observed for the induced magnetic field. Figure 7 shows that the velocity profile notably decreases for higher values of the thermal radiation parameter, whereas in Fig. 8 the induced magnetic field shows slight increments. Figures 9 and 10 represent the profiles of velocity and the induced magnetic field for the Prandtl number for air $(\operatorname{Pr}=.71)$ and water( $\operatorname{Pr}=7)$. It shows that velocity decreases as Pr increases while the induced magnetic field shows an adverse effect. The variations of the velocity profile and induced magnetic profile for different values of the magnetic field $(M)$ are shown in Figs 11 and 12 .With an increase in $M$ the flow is decelerated, i.e., velocity decreases. The behavior of $M$ shows that the applied magnetic field $H_{0}$, is therefore effectively moving with the free stream. On the other hand, in all the cases, the induced magnetic field decreased for increasing values of $M$.

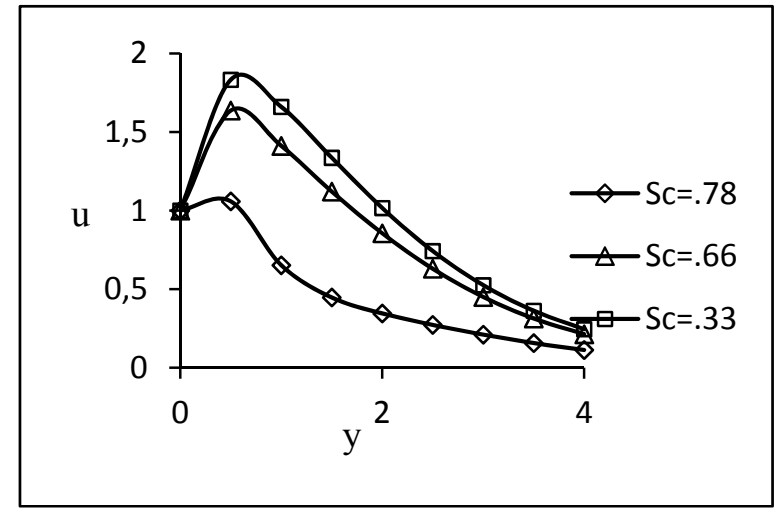

Fig.3. Effect of Sc on velocity.



Fig.4. Effect of Sc on induced magnetic field. 


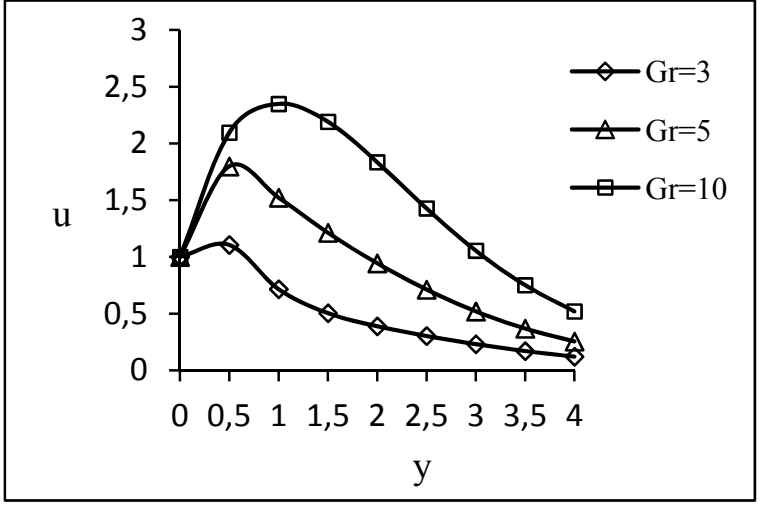

Fig.5. Effect of Gr on velocity.

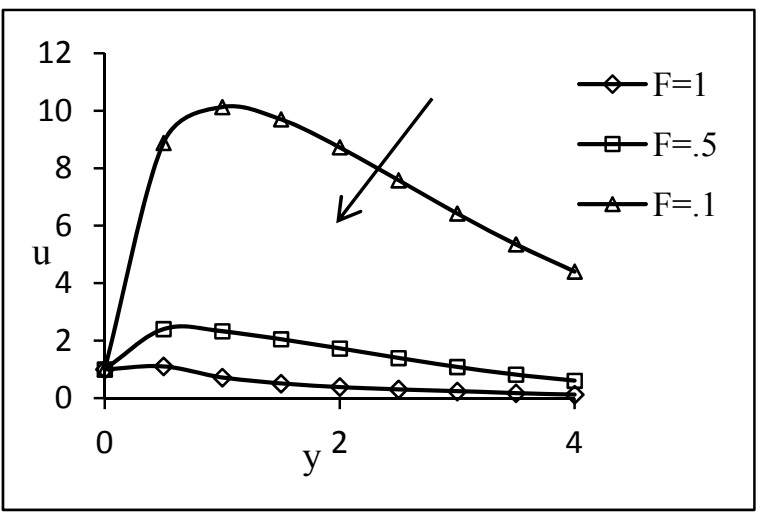

Fig.7. Effect of $F$ on velocity.

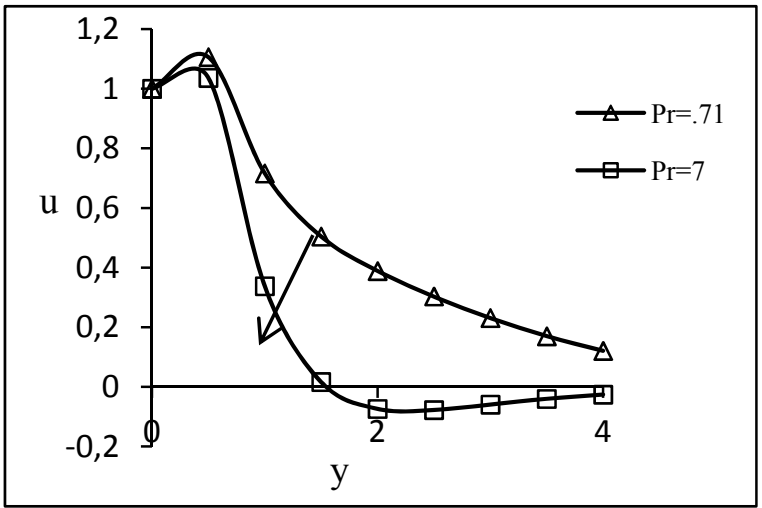

Fig.9. Effect of Pr on velocity.

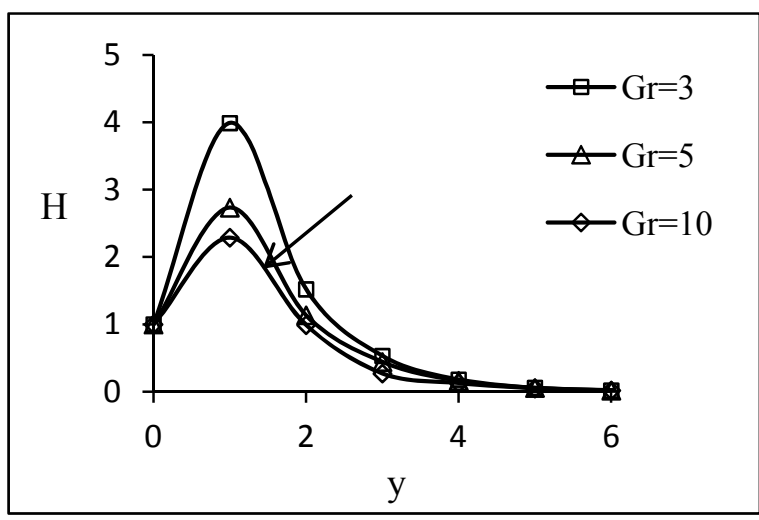

Fig.6. Effect of Gr on induced magnetic field.

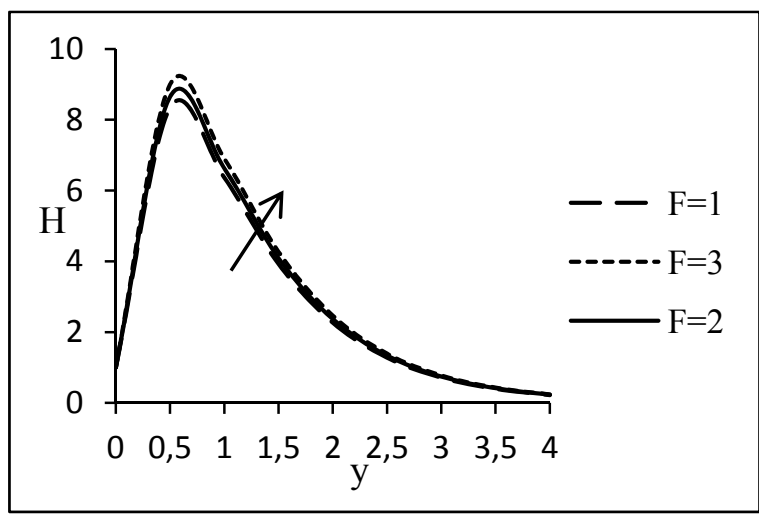

Fig.8. Effect of radiation $F$ on induced magnetic field.

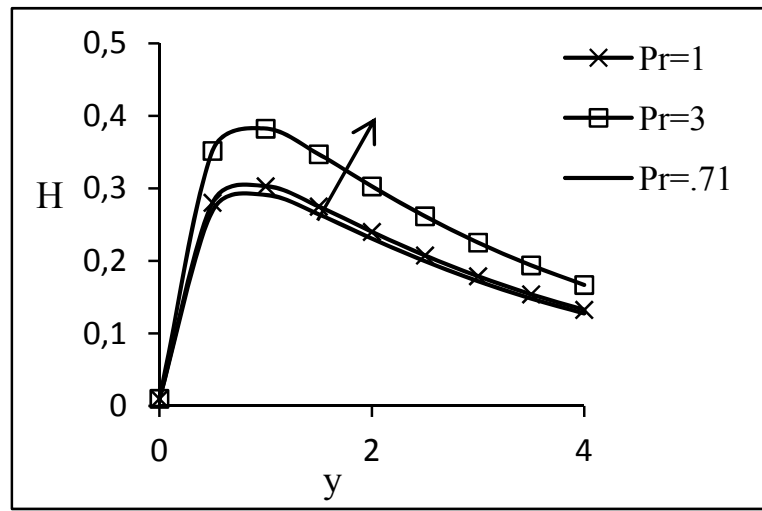

Fig.10. Effect of Pr on induced magnetic field. 




Fig.11. Effect Hartmann number M on velocity.

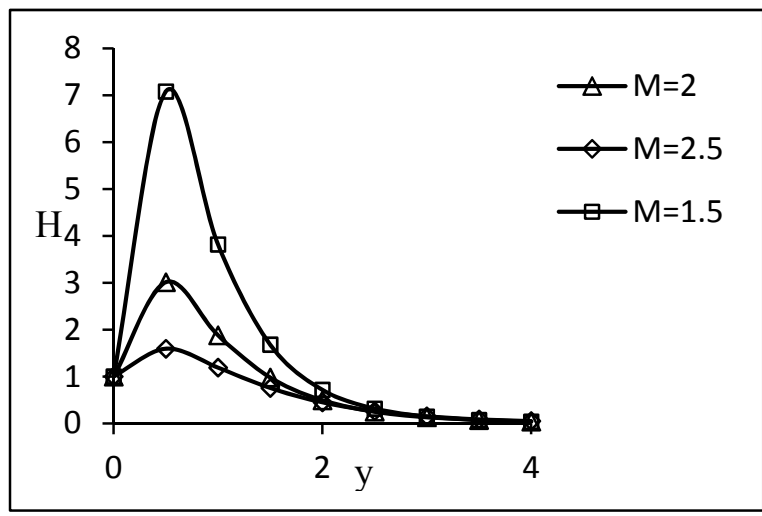

Fig.12. Effect of $M$ on induced magnetic field.

Figures 13 and 14 show the effect of various parameters on $H$ and $u$, when thermal radiation and radiation absorption are not taken into account. It is observed that the results are in good agreement with those of Chaudhary and Sharma [12].

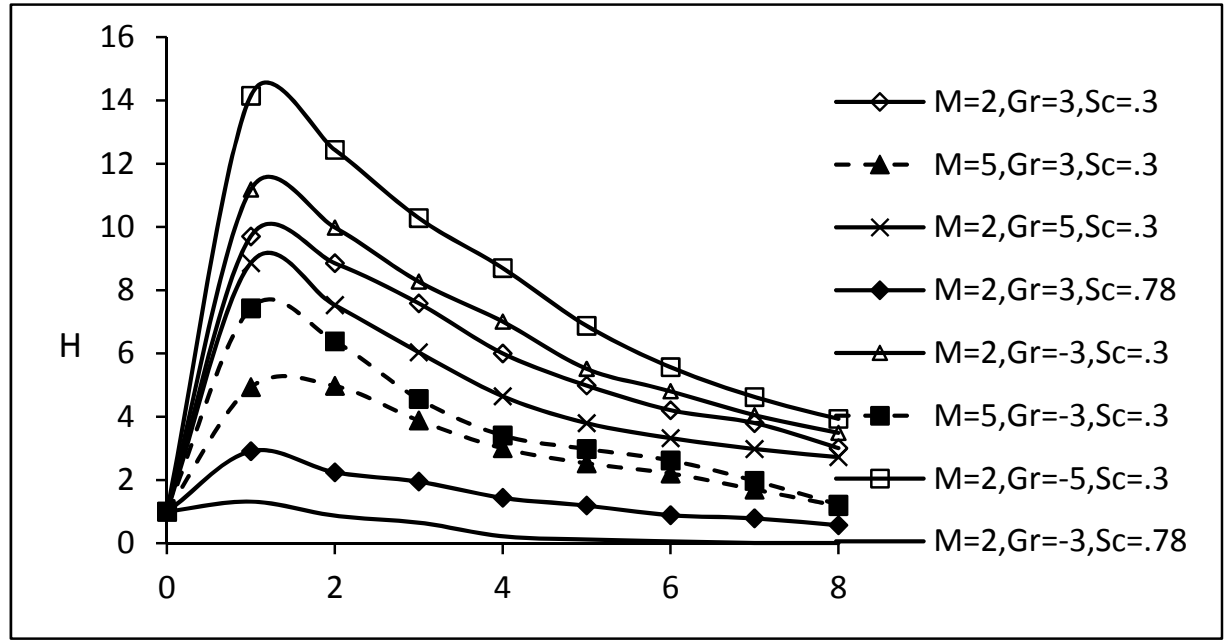

Fig.13. Effect of M, Gr and Sc on $H$ when $F=0, \lambda=0, \alpha_{c}=0$.

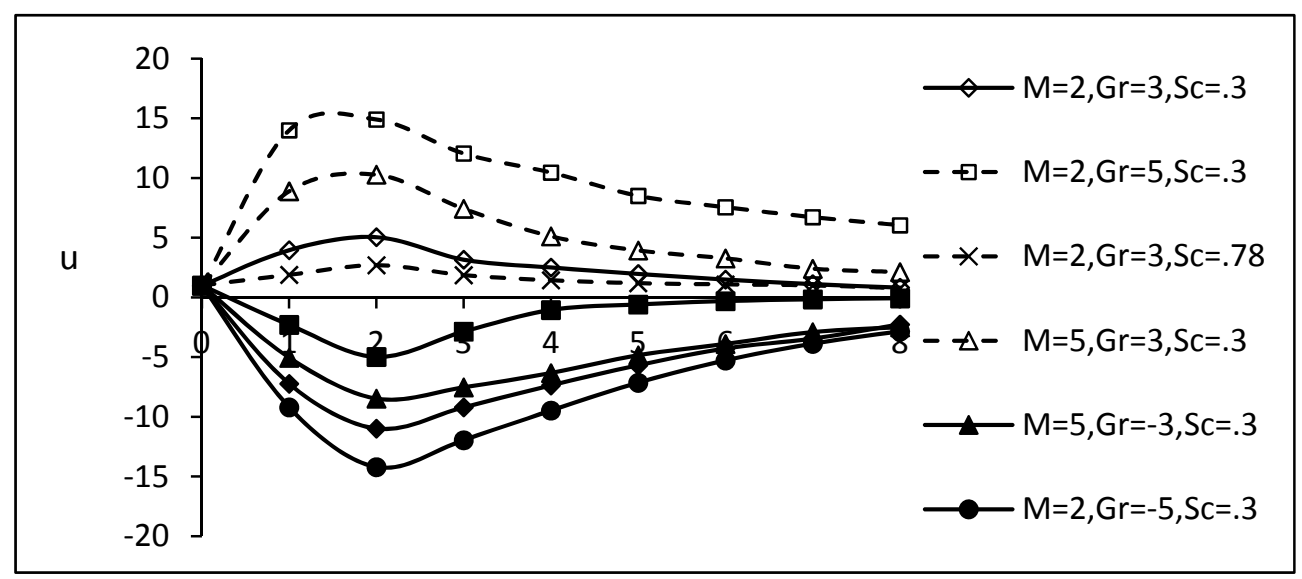

Fig.14. Effect of M, Gr and Sc on $u$ when $F=0, \lambda=0, \alpha_{c}=0$. 
Figures 15-21 represent the effect of $\mathrm{Bi}, \lambda, \alpha_{c}, \mathrm{Pr}, \mathrm{Sc}, \mathrm{Gr}, F$ and $M$ on temperature distributions. It is clear from Fig. 15 that an increase in the radiation absorption parameter, increases the heat transfer. The Biot number is the ratio of internal thermal resistance of the channel to the boundary layer thermal resistance. When $\mathrm{Bi}=0$ (i.e., without the Biot number) the inside of the channel with the hot fluid is totally insulated, the internal thermal resistance of the channel is extremely high and no convective heat transfer to the cold fluid on the outside of the channel takes place. Moreover, it is noted that the fluid temperature decreases for increasing the Biot number, since as Bi increases, the thermal resistance of the plate decreases and convective heat transfer to the fluid increases. Figures 16, 17 show the influence of the Prandtl number and Schmidt number on temperature. It is noticed that for higher values of $\mathrm{Pr}$ and Sc temperature falls to the boundary layer and tends to zero. The reason is that smaller values of Pr are equivalent to increasing the thermal conductivities, and therefore heat is able to diffuse away from the heated plate more rapidly than for higher values of Pr. Figure 18 shows that temperature increases as the thermal Grashof number increases. Figures 19, 20, 21 show the impact of $\lambda, M$ and $F$ on the temperature field, respectively. Figure 21 represents that an increase in the radiation parameter decreases the temperature distribution because large values of the radiation parameter enhance the conduction over radiation, thereby the thickness of the thermal boundary layer is decreased. It is observed from Fig. 22 that we obtain a destructive type concentration profile for the chemical reaction because the concentration decreases by increasing the chemical reaction parameter which indicates that the diffusion rates can be changed by the chemical reaction parameter. Figure 23 elucidates that an increase in the Schmidt number increases the conduction which decreases the mass transfer. Moreover, as compared to the case of no chemical reaction $(\lambda=0)$, the concentration profile we get for the Schmidt number as expected by Chaudhary and Bhupendra [12].

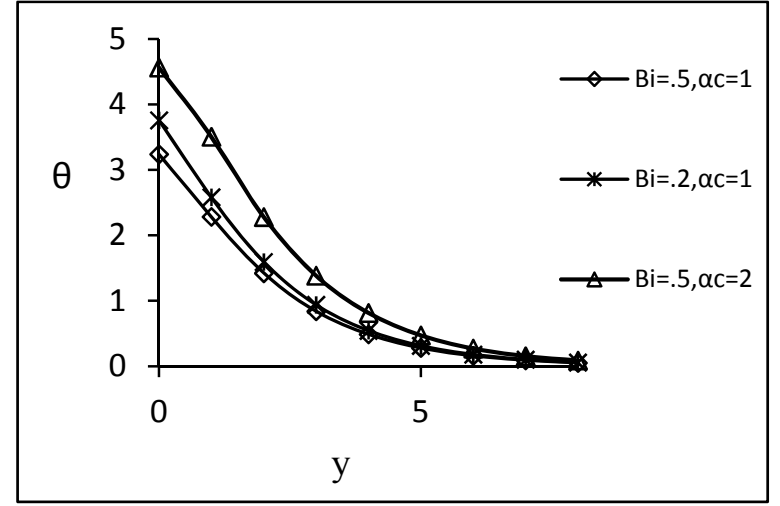

Fig.15. Effect of Biot number Bi on temperature.

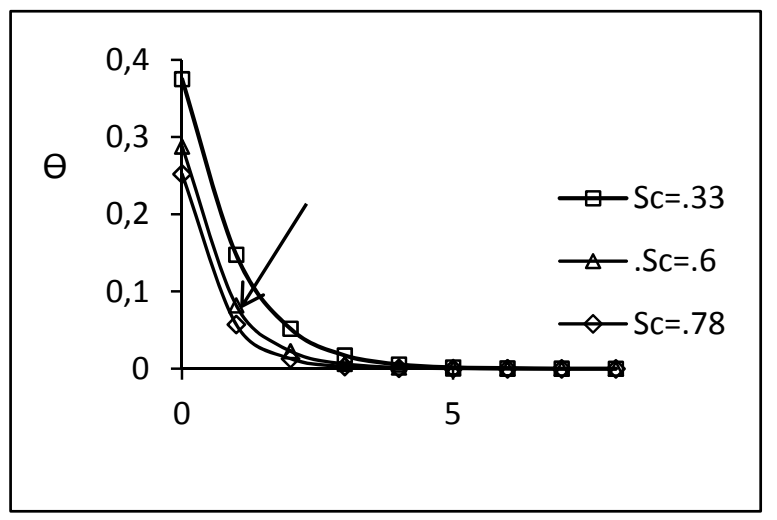

Fig.17. Effect of Sc on temperature.

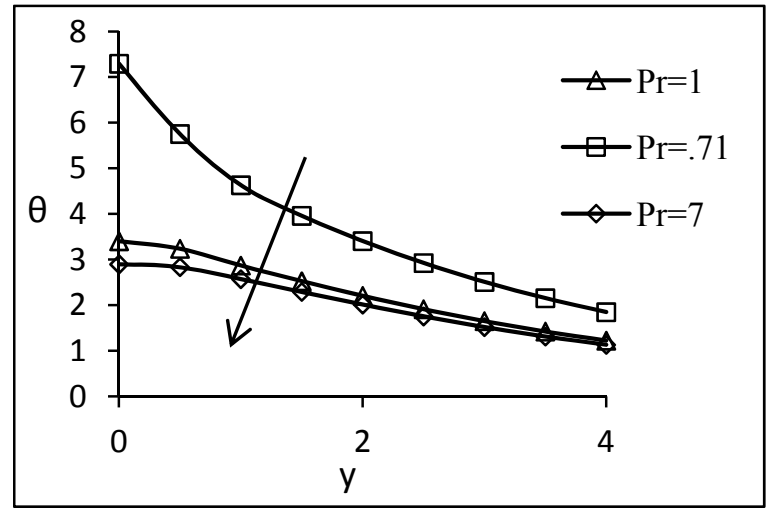

Fig.16. Effect of Pr on temperature.



Fig.18. Effect of Gr on temperature. 


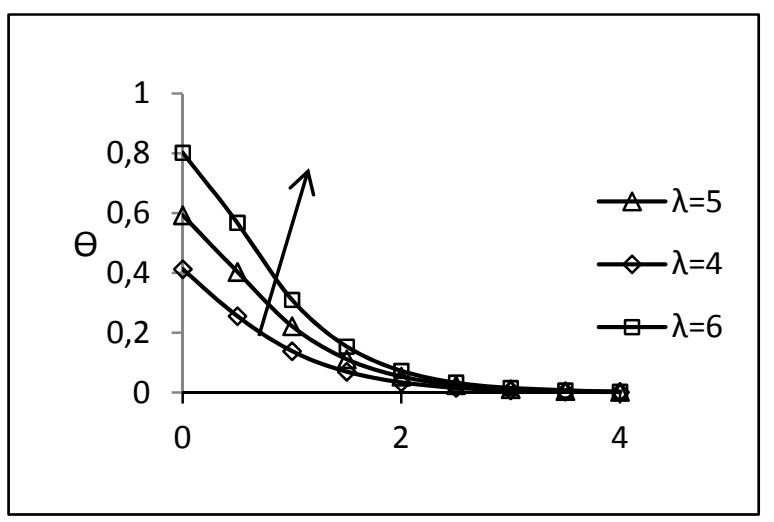

Fig.19. Effect of $\lambda$ on temperature.

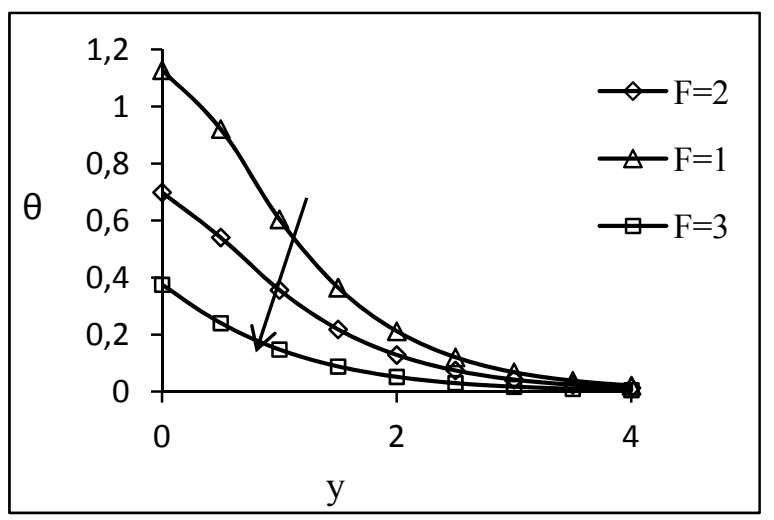

Fig.21. Effect of $F$ on temperature.



Fig.23. Effect of Sc on concentration.

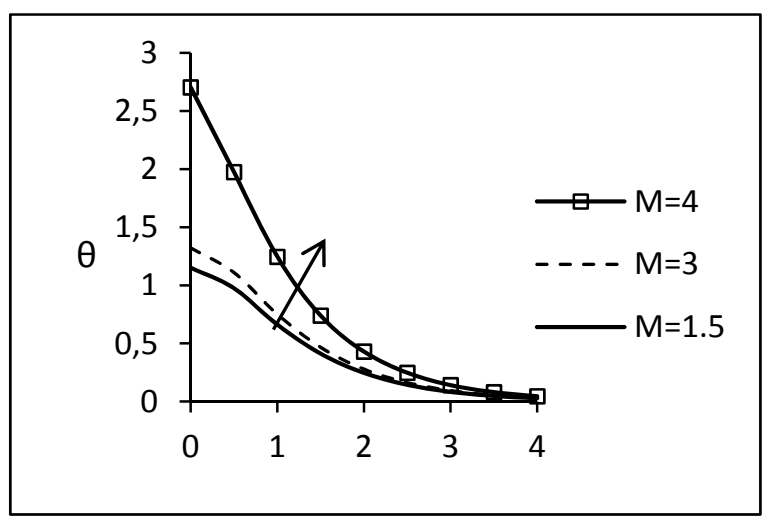

Fig.20. Effect of M on temperature.

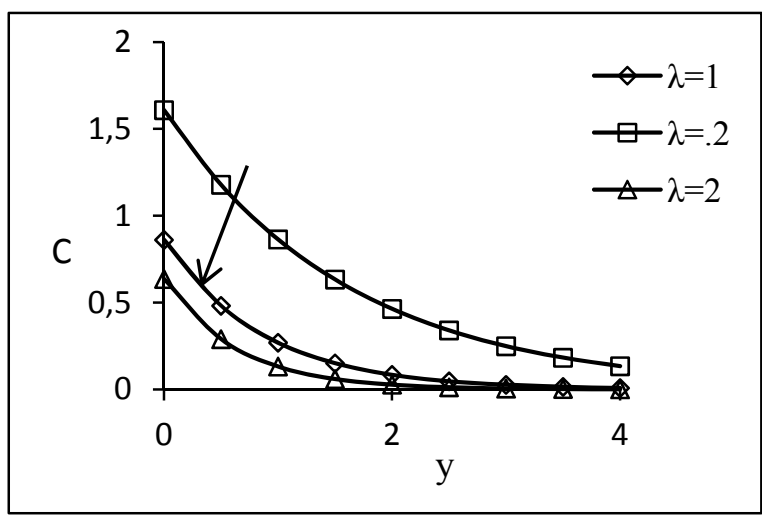

Fig.22. Effect of $\lambda$ on concentration

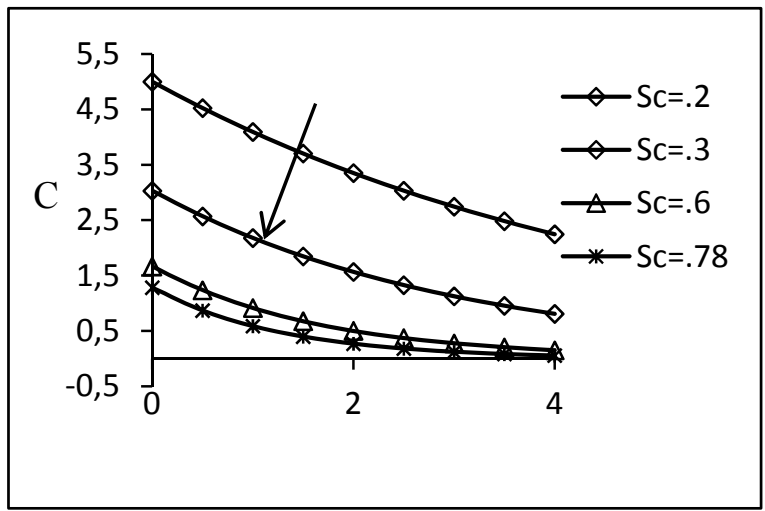

Fig.24. Effect of Sc on concentration when $\lambda=0$.

The current density $J$ has been presented against $y$ in Figs 25-27 for air $(\operatorname{Pr}=.71)$ for different values of $F, \operatorname{Sc}, \operatorname{Pr}, \alpha_{c}, \lambda, h, M$ and Bi. In Fig. 25 it is noted that current density initially increases as radiation increases, then starts decreasing. Further, a decreasing value of Sc decreases the current density. Figure 26 shows that current density decreases as Pr increases while it shows reverse effect for $\lambda$ and $\alpha_{c . .}$ The influence of $h$ and $M$ is shown in Fig.27; when $h$ increases current density decreases but destructive profile shows for higher values of $M$. Further, the effect of the Biot number is displayed in Fig.28. It can be seen that current density decreases as Bi increases. The effect of radiation on the Nusselt number is presented in Fig.29. It is shown that an increased radiation parameter decreases the 
rate of heat transfer. Further the effect of the Biot number on skin friction is shown in Fig.30. It can be seen that skin friction decreases as Bi increases.

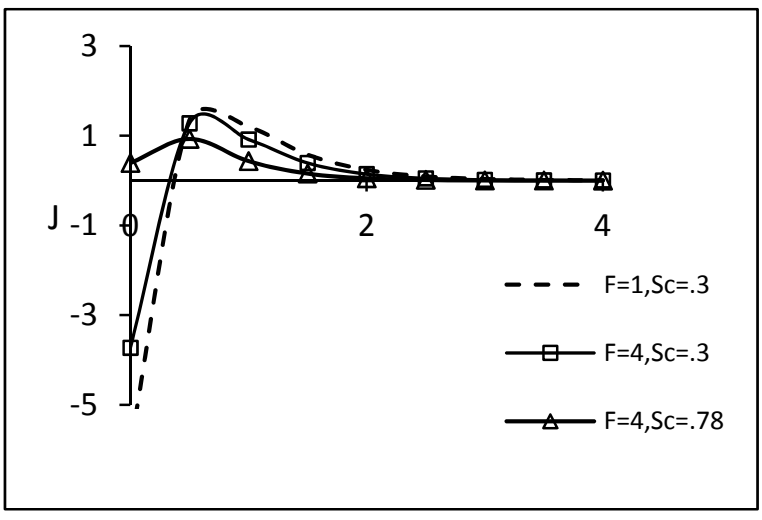

Fig.25. Effect of $F$ and Sc on current density.

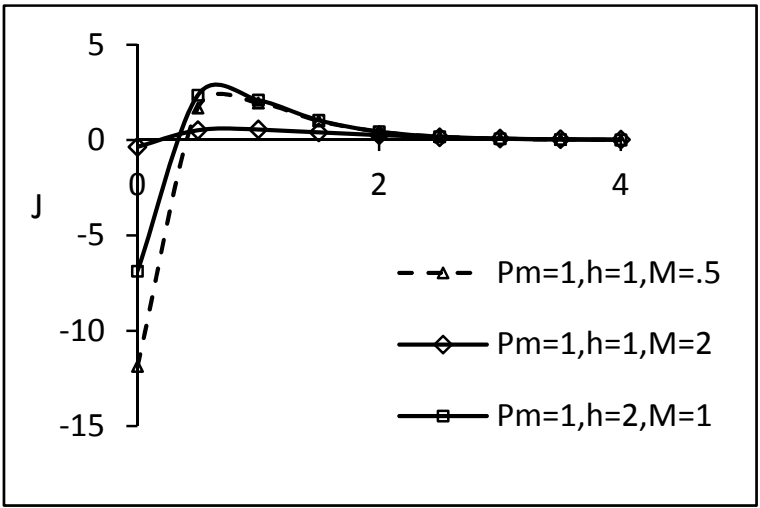

Fig.27. Effect of $P m, h$ and $\mathrm{M}$ on current density.

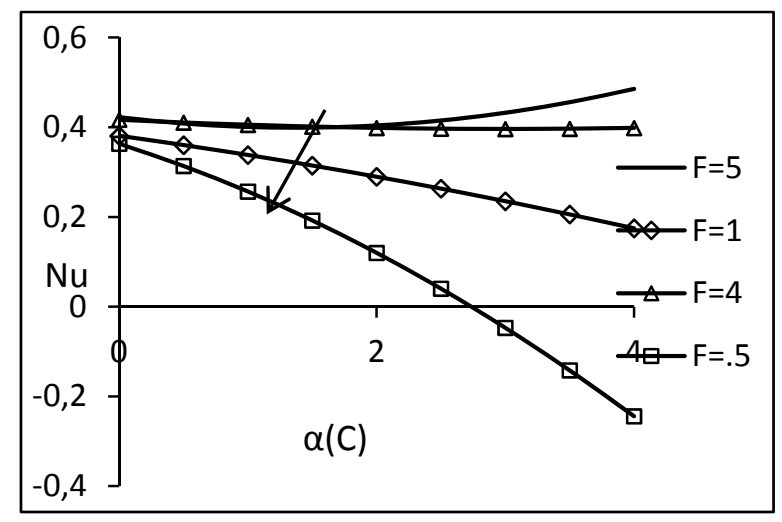

Fig.29. Effect of $F$ on Nusselt number.

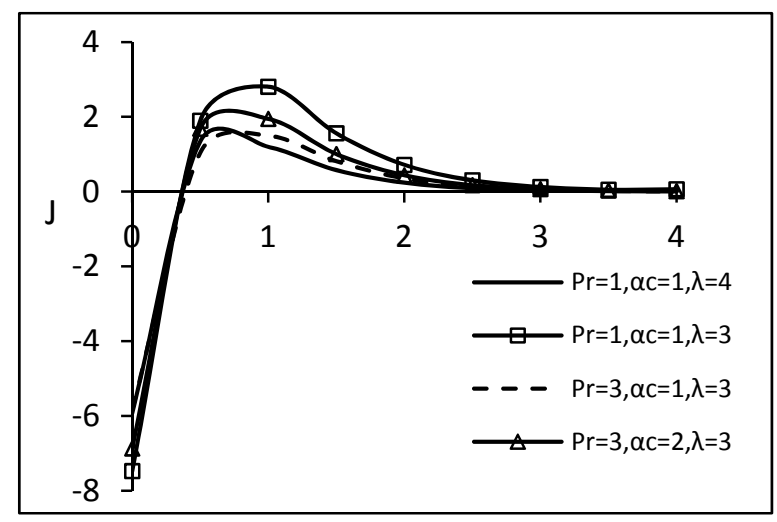

Fig.26. Effect of $\operatorname{Pr}, \alpha_{c}$ and $\lambda$ on current density.

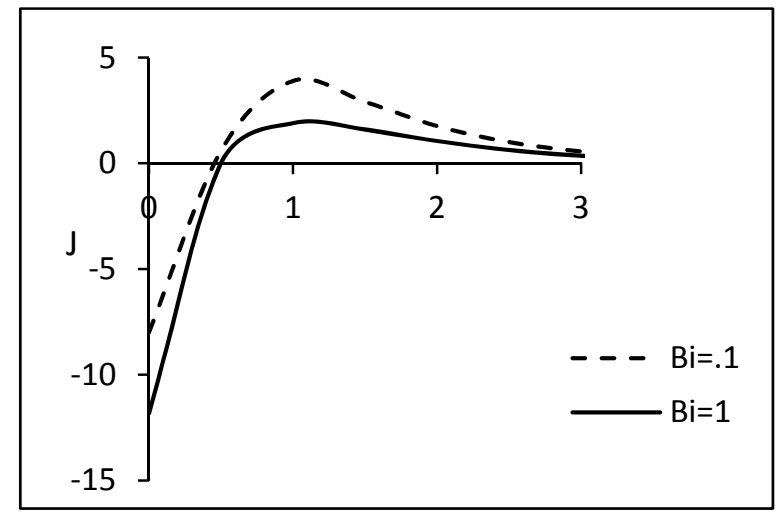

Fig.28. Effect of Bi on current density.

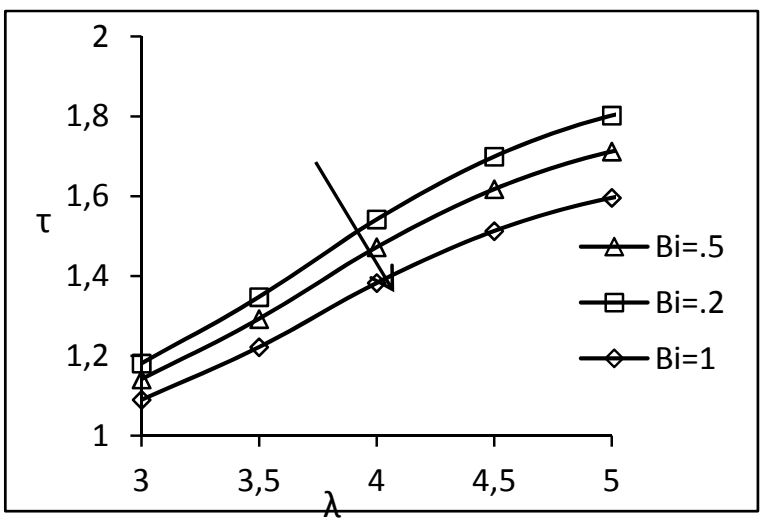

Fig.30. Effect of Bi on skin friction. 


\section{Nomenclature}

$B$ - coefficient of volume expansion for heat transfer

$\mathrm{Bi}$ - Biot number

$C_{P}$ - specific heat at constant pressure

Ec - Eckert number/dissipative heat

$F$ - radiation parameter

$G$ - acceleration due to gravity

$\mathrm{Gr}$ - thermal Grashof number

$H$ - induced magnetic field

$H_{0}$ - uniform magnetic field

$H_{x}$ - induced magnetic field along $x$-direction

$J$ - current density

M - Hartmann number

$N$ - kinematic viscosity

$P$ - density

Pm - magnetic Prandtl number

Pr - Prandtl number

$T$ - temperature

$T_{W}$ - fluid temperature at the surface

$T_{\infty} \quad$ - fluid temperature in the free stream

$U$ - velocity component in $x$-direction

$U_{0}$ - dimensionless free stream velocity

$Q_{C}$ - radiation absorption parameter

$q_{r}$ - radiative heat flux

$V_{0}$ - suction velocity

$\alpha_{c}$ - radiation absorption parameter

$\Theta$ - dimensionless fluid temperature

$\kappa-$ thermal conductivity

$\mu_{0}-$ magnetic diffusivity

$\Sigma$ - Stefan-Boltzmann constant

\section{Appendix}

$$
\begin{aligned}
& A_{2}=\frac{\alpha_{C}}{m_{1}\left(m_{1}^{2}-\operatorname{Pr} m_{1}-F\right)}, \quad A_{1}=\frac{\left[B_{i}-A_{2}\left(m_{1}+B_{i}\right)\right]}{m_{2}+B_{i}}, \\
& b_{2}=\frac{\operatorname{GrPm} M A_{2}}{-m_{1}^{3}+(P m+1) m_{1}^{2}-P m\left(1-M^{2}\right) m_{1}}, \quad b_{3}=\frac{G c \operatorname{Pm} M}{-m_{1}^{3}+(P m+1) m_{1}^{2}-P m\left(1-M^{2}\right) m_{1}}, \\
& A_{4}=\frac{G r \operatorname{Pm} A_{1}}{-m_{2}^{3}+(P m+1) m_{2}^{2}-P m\left(1-M^{2}\right) m_{2}}, \quad A_{3}=b_{2}+b_{3}, \quad m_{2}=\frac{\operatorname{Pr}+\sqrt{\operatorname{Pr}^{2}+4 F}}{2}, \\
& m_{2}=\frac{\operatorname{Sc}+\sqrt{\mathrm{Sc}^{2}+4 \lambda}}{2}, \quad \frac{(\operatorname{Pm}+1)+\sqrt{(\operatorname{Pm}+1)^{2}-4 \operatorname{Pm}\left(1-M^{2}\right)}}{2}, \quad A_{6}=h-\left(A_{3}+A_{4}\right),
\end{aligned}
$$




$$
\begin{aligned}
& A_{7}=\frac{\mathrm{Gr} A_{1}+A_{4} m_{2} \mathrm{M}}{m_{2}^{2}-m_{2}}, \quad A_{8}=\frac{-\mathrm{Gr} A_{2}+A_{3} m_{1} \mathrm{M}-\frac{G m}{m_{1}}}{m_{2}^{2}-m_{2}}, \quad A_{9}=\frac{A_{6} m_{3} \mathrm{M}}{m_{3}^{2}-m_{3}}, \\
& A_{10}=u_{0}-\left(A_{7}+A_{8}+A_{9}\right) \\
& b_{4}=-\operatorname{Pr} m_{2}^{2} A_{7}^{2}-\frac{\operatorname{Pr}}{\operatorname{Pm}} A_{4}^{2} m_{2}^{2}, \quad b_{5}=-\operatorname{Pr} m_{1}^{2} A_{8}^{2}-\frac{\operatorname{Pr}}{\operatorname{Pm}} A_{3}^{2} m_{1}^{2}, \\
& b_{6}=-\operatorname{Pr} m_{3}^{2} A_{9}^{2}-\frac{\operatorname{Pr}}{\operatorname{Pm}} A_{6}^{2} m_{3}^{2}, \quad b_{7}=-\operatorname{Pr} 2 m_{2} A_{7} m_{1} A_{8}-\frac{\operatorname{Pr}}{\operatorname{Pm}} 2 m_{2} A_{3} m_{1} A_{4}, \\
& b_{8}=-\operatorname{Pr} 2 m_{2} A_{7} m_{3} A_{9}-\frac{\operatorname{Pr}}{\operatorname{Pm}} 2 m_{2} A_{6} m_{3} A_{4}, \quad b_{9}=-\operatorname{Pr} 2 m_{3} A_{9} m_{1} A_{8}-\frac{\operatorname{Pr}}{\operatorname{Pm}} 2 m_{3} A_{3} m_{1} A_{6}, \\
& A_{11}=\frac{b_{4}}{4 m_{2}^{2}-2 \operatorname{Pr} m_{2}-F}, \quad A_{12}=\frac{b_{5}}{4 m_{1}^{2}-2 \operatorname{Pr} m_{1}-F}, \quad A_{13}=\frac{b_{6}}{4 m_{3}^{2}-2 \operatorname{Pr} m_{3}-F}, \\
& A_{14}=\frac{b_{7}}{\left(m_{1}+m_{2}\right)^{2}-\operatorname{Pr}\left(m_{1}+m_{2}\right)-F}, \quad A_{15}=\frac{b_{8}}{\left(m_{3}+m_{2}\right)^{2}-\operatorname{Pr}\left(m_{3}+m_{2}\right)-F}, \\
& A_{16}=\frac{b_{9}}{\left(m_{1}+m_{3}\right)^{2}-\operatorname{Pr}\left(m_{1}+m_{3}\right)-F}, \quad A_{17}=\frac{-A_{10}^{2} \operatorname{Pr}}{4-2 \operatorname{Pr}-F}, \\
& A_{18}=\frac{-2 \operatorname{Pr} m_{2} A_{10} A_{7}}{\left(1+m_{2}\right)^{2}-\operatorname{Pr}\left(1+m_{2}\right)-F}, \quad A_{19}=\frac{-2 \operatorname{Pr} m_{1} A_{10} A_{8}}{\left(1+m_{1}\right)^{2}-\operatorname{Pr}\left(1+m_{1}\right)-F}, \\
& A_{20}=\frac{-2 \operatorname{Pr} m_{3} A_{10} A_{9}}{\left(1+m_{3}\right)^{2}-\operatorname{Pr}\left(1+m_{3}\right)-F}, \quad b_{10}=\frac{A_{21} \mathrm{Gr} \operatorname{PmM}}{-m_{2}}, \\
& b_{11}=\frac{A_{11} \mathrm{GrPmM}}{-2 m_{2}}, \quad b_{12}=\frac{A_{12} \mathrm{GrPmM}}{-2 m_{1}}, \quad b_{13}=\frac{A_{13} \mathrm{GrPmM}}{-2 m_{3}}, \quad b_{14}=\frac{A_{14} \mathrm{GrPmM}}{-\left(m_{1}+m_{2}\right)}, \\
& b_{15}=\frac{A_{15} \mathrm{GrPmM}}{-\left(m_{3}+m_{2}\right)}, \quad b_{16}=\frac{A_{16} G r P m M}{-\left(m_{1}+m_{3}\right)}, \quad b_{17}=\frac{A_{17} \mathrm{GrPm} \mathrm{M}}{-2}, \\
& b_{18}=\frac{A_{18} \mathrm{GrPmM}}{-\left(1+m_{2}\right)}, \quad b_{19}=\frac{A_{19} \mathrm{GrPmM}}{-\left(m_{1}+1\right)}, \quad b_{20}=\frac{A_{20} \mathrm{GrPm} \mathrm{M}}{-\left(m_{3}+1\right)}, \\
& A_{22}=\frac{b_{10}}{m_{2}^{2}-m_{2}(1+\operatorname{Pm})+\operatorname{Pm}\left(1-M^{2}\right)}
\end{aligned}
$$




$$
\begin{aligned}
& A_{23}=\frac{b_{11}}{4 m_{2}^{2}-2 m_{2}(1+\operatorname{Pm})+\operatorname{Pm}\left(1-M^{2}\right)}, \quad A_{24}=\frac{b_{12}}{4 m_{1}^{2}-2 m_{l}(1+\operatorname{Pm})+\operatorname{Pm}\left(1-M^{2}\right)}, \\
& A_{25}=\frac{b_{13}}{4 m_{3}^{2}-2 m_{3}(1+\operatorname{Pm})+\operatorname{Pm}\left(1-M^{2}\right)}, \\
& A_{26}=\frac{b_{14}}{\left(m_{1}+m_{2}\right)^{2}-\left(m_{1}+m_{2}\right)(1+\operatorname{Pm})+\operatorname{Pm}\left(1-M^{2}\right)}, \\
& A_{27}=\frac{b_{15}}{\left(m_{3}+m_{2}\right)^{2}-\left(m_{3}+m_{2}\right)(1+\operatorname{Pm})+\operatorname{Pm}\left(1-M^{2}\right)}, \\
& A_{28}=\frac{b_{16}}{\left(m_{3}+m_{1}\right)^{2}-\left(m_{3}+m_{l}\right)(1+\operatorname{Pm})+\operatorname{Pm}\left(1-M^{2}\right)}, \\
& A_{29}=\frac{b_{17}}{4-2(1+\operatorname{Pm})+\operatorname{Pm}\left(1-M^{2}\right)}, \quad A_{30}=\frac{b_{18}}{\left(1+m_{2}\right)^{2}-\left(1+m_{2}\right)(1+\operatorname{Pm})+\operatorname{Pm}\left(1-M^{2}\right)}, \\
& A_{31}=\frac{b_{19}}{\left(1+m_{1}\right)^{2}-\left(1+m_{1}\right)(1+\operatorname{Pm})+\operatorname{Pm}\left(1-M^{2}\right)}, \\
& A_{32}=\frac{b_{20}}{\left(1+m_{3}\right)^{2}-\left(1+m_{3}\right)(1+\operatorname{Pm})+\operatorname{Pm}\left(1-M^{2}\right)}, \\
& A_{33}=-\left(A_{22}+A_{23}+A_{24}+A_{25}+A_{26}+A_{27}+A_{28}+A_{29}+A_{30}+A_{31}+A_{32}\right), \\
& X_{1}=A_{11}\left(2 m_{2}+B_{i}\right)+A_{12}\left(2 m_{1}+B_{i}\right)+A_{13}\left(2 m_{3}+B_{i}\right)+A_{14}\left[\left(m_{2}+m_{1}\right)+B_{i}\right]+ \\
& +A_{15}\left[\left(m_{2}+m_{3}\right)+B_{i}\right]+A_{16}\left[\left(m_{3}+m_{1}\right)+B_{i}\right]+A_{17}\left[2+B_{i}\right]+A_{18}\left[\left(m_{2}+1\right)+B_{i}\right]+ \\
& +A_{19}\left[\left(m_{1}+1\right)+B_{i}\right]+A_{20}\left[\left(m_{3}+1\right)+B_{i}\right] \text {, } \\
& A_{21}=-\frac{X_{1}}{\left(m_{2}+B_{i}\right)}, \quad A_{33 a}=\frac{-\mathrm{Gr} A_{21}+A_{22} \mathrm{M} m_{2}}{m_{2}^{2}-m_{2}}, \quad A_{34}=\frac{-\mathrm{Gr} A_{11}+2 A_{23} \mathrm{M} m_{2}}{4 m_{2}^{2}-2 m_{2}}, \\
& A_{35}=\frac{-\mathrm{Gr} A_{12}+2 A_{24} \mathrm{M} m_{1}}{4 m_{1}^{2}-2 m_{1}}, A_{36}=\frac{-\mathrm{Gr} A_{13}+2 A_{25} \mathrm{M} m_{3}}{4 m_{3}^{2}-2 m_{3}}, A_{37}=\frac{-\mathrm{Gr} A_{14}+A_{26} \mathrm{M}\left(m_{1}+m_{2}\right)}{\left(m_{1}+m_{2}\right)^{2}-\left(m_{1}+m_{2}\right)}, \\
& A_{38}=\frac{-\mathrm{Gr} A_{15}+A_{27} \mathrm{M}\left(m_{3}+m_{2}\right)}{\left(m_{3}+m_{2}\right)^{2}-\left(m_{3}+m_{2}\right)}, \quad A_{39}=\frac{-\mathrm{Gr} A_{16}+A_{28} \mathrm{M}\left(m_{1}+m_{3}\right)}{\left(m_{1}+m_{3}\right)^{2}-\left(m_{1}+m_{3}\right)},
\end{aligned}
$$




$$
\begin{aligned}
& A_{40}=\frac{-\mathrm{Gr} A_{17}+2 A_{29} \mathrm{M}}{2}, \quad A_{41}=\frac{-\mathrm{Gr} A_{18}+A_{30} \mathrm{M}\left(1+m_{2}\right)}{\left(1+m_{2}\right)^{2}-\left(1+m_{2}\right)}, A_{42}=\frac{-\mathrm{Gr} A_{19}+A_{31} \mathrm{M}\left(1+m_{1}\right)}{\left(1+m_{1}\right)^{2}-\left(1+m_{1}\right)}, \\
& A_{41}=\frac{-\mathrm{Gr} A_{20}+A_{32} \mathrm{M}\left(1+m_{3}\right)}{\left(1+m_{3}\right)^{2}-\left(1+m_{3}\right)}, \quad A_{44}=\frac{\mathrm{M} m_{3} A_{33}}{m_{3}^{2}-m_{3}}, \\
& A_{33}=-\left(A_{33 a}+A_{34}+A_{35}+A_{36}+A_{37}+A_{38}+A_{39}+A_{40}+A_{41}+A_{42}+A_{43}+A_{44}\right), \\
& \theta_{1}=A_{1} e^{-m_{2} y}+A_{2} e^{-m_{1} y}, \quad H_{1}=A_{6} e^{-m_{3} y}+A_{3} e^{-m_{1} y}+A_{4} e^{-m_{2} y}, \\
& u_{1}=A_{10} e^{-y}+A_{9} e^{-m_{3} y}+A_{8} e^{-m_{1} y}+A_{7} e^{-m_{2} y}, \\
& A_{42} e^{-\left(1+m_{1}\right) y}+A_{43} e^{-\left(1+m_{3}\right) y}+A_{44} e^{-m_{3} y} .
\end{aligned}
$$

\section{References}

[1]. Soundalgekar V.M. (1974): Free convection effects on steady MHD flow past a vertical porous plate. - J. Fluid Mechanics, vol.66, pp.541-551.

[2]. Soundalgekar V.M. (1975): Free convection effects on the oscillatory flow of an incompressible, electrically conducting, viscous fluid past an infinite, vertical porous plate with constant suction and the transverse magnetic field. - Zeitschrift für Angewandte Mathematik und Mechanik (ZAMM), vol.55, No.5, pp.257-267.

[3]. Kafousias N.G. and Georgantopoulos G.A. (1982): Magnetohydrodynamic free convection effects on the Stokes problem for an incompressible viscous fluid past an infinite vertical limiting surface. - Astrophysics and Space Science, vol.85, pp.297-307.

[4]. Raptis A. and Kafoussias N.G. (1982): Magnetohydrodynamic free convection flow and mass transfer through porous medium bounded by an infinite vertical porous plate with constant. - Canadian Journal of Physics, vol.60, No.12, pp.1725-1729.

[5]. Ramana Kumari C.V. and Bhaskara Reddy N. (1994): Mass transfer effects on unsteady free convective flow past an infinite vertical porous plate with variable suction. - J. Energy Heat Mass Transfer, vol.16, pp.279-287.

[6]. Elbashbeshy E.M. (2003): The mixed convection along a vertical plate embedded in non-Darcian porous medium with suction and injection. - J. Appl. Math. Comput., vol.136, No.1, pp.139-149.

[7]. Makinde O.D. (2005): Free convection flow with thermal radiation and mass transfer past a moving vertical porous plate. - Int. Commun. Heat Mass Transfer, pp.1411-1419.

[8]. Alam M.S., Rahman M.M. and Samad M.A. (2009): Transient magnetohydrodynamic free convective heat and mass transfer flow with thermophoresis past a radiate inclined permeable plate in the presence of variable chemical reaction and temperature dependent viscosity. - Nonlinear Anal. Model. Control, vol.14, pp.3-20.

[9]. Ahmed S. (2010): Free and forced convective MHD oscillatory flow over an infinite porous surface in an oscillatory free stream. - Latin American Applied Research, vol.40, pp.167-173.

[10]. Gireesh Kumar J. and Satyanarayana P.V. (2011): Mass transfer effect on MHD unsteady free convective Walters's memory flow with constant suction and heat sink. - Int. J. Appl. Math. Mech., vol.19, pp.97-109.

[11]. Megakhed A.A. (1974): Effect of induced magnetic field and heat transfer on non-stationary magneto hydrodynamic flow around a porous plate. - Magnetohydrodynamics, vol.10, No.1, pp.48-52. 
[12]. Chaudhary R.C. and Bhupendra K.S. (2006): Combined heat and mass transfer by laminar mixed convection flow from a vertical surface with induced magnetic field. - J. Appl. Phys., vol.99, 349.

[13]. Md. Alom S. Rahman M.M. and Md. A. Sattar (2007): Similarity solutions for hydromagnetic free convective heat and mass transfer flow along a semi-infinite permeable inclined flat plate with heat generation and thermophoresis. - Nonlinear Analysis Modelling and Control, vol.12, No.4, pp.433-445.

[14]. Alom M.M., Rafiqul I.M. and Rahman F. (2008): Steady heat and mass transfer by mixed convection flow from a vertical porous plate with induced magnetic field, constant heat and mass fluxes. - Thammasat. Int. J. Sc. Tech., vol.13, No.4, pp.1-13.

[15]. Beg O.A., Bakier A.Y., Prasad V.R., Zueco J. and Ghosh S.K. (2009): Non similar, laminar, steady, electricallyconducting forced convection liquid metal boundary layer flow with induced magnetic field effects. - Int. J. Ther. Sci., vol.48, pp.1596-1606.

[16]. Ghosh S.K., Bég O.A. and Zueco J. (2009): Hydromagnetic free convection flow with induced magnetic field effects. - Physics and Astronomy, vol.45, No.2, pp.175-185.

[17]. Ahmed S. (2010): Induced magnetic field with radiating fluid over a porous vertical plate analytical study. - J. Nav. Archi. Marine. Eng., vol.7, No.2, pp.83-94.

[18]. Ahmed S. (2012): Mathematical model of induced magnetic field with viscous/magnetic dissipation bounded by a porous vertical plate in the presence of radiation. - Int. J. Appl. Math. Mech., vol.8, No.1, pp.86-104.

[19]. Batler R.C. (2008): Radiation effects for the Blassius and Sakiadis flows with a convective surface boundary condition. - Appl. Math. Comput., vol.20, No.6, pp.832-840.

[20]. Makinde O.D. and Aziz A. (2010): MHD mixed convection from a vertical plate embedded in a porous medium with a convective boundary condition. - Int. J. Therm. Sci., vol.49, pp.1813-20.

[21]. Yao S., Fang T. and Zhong Y. (2011): Heat transfer of a generalized stretching/shrinking wall problem with convective boundary conditions. - Commun. Nonlin. Sci. Numer. Simulat., vol.16, pp.752-760.

[22]. Sivraj R. and Rushi Kumar B. (2012): Chemically reacting dusty viscoelastic fluid flow in an irregular channel with convective boundary. - Ain Shams Engineering Journal, vol.4, pp.93-101.

[23]. Hossain M.A. and Takhar H.S. (1996): Radiation effect on mixed convection along a vertical plate with uniform surface temperature. - J. Heat Mass Transfer, vol.31, pp.243-248.

[24]. Raptis A. and Massalas C.V. (1998): Magnetohydrodynamic flow past a plate by the presence of radiation. - Heat Mass Transfer, vol.34, pp.107-109.

[25]. Raptis A. and Perdikis C. (2003): Thermal radiation of an optically thin gray gas. - Int. J. Appl. Mech. Eng., vol.8, pp.131-134.

[26]. Raptis A., Perdikis C. and Leontitsis A. (2003): Effects of radiation in an optically thin gray gas flowing past a vertical infinite plate in the presence of a magnetic field. - Heat and Mass Transfer, vol.39, pp.771-773.

[27]. Raptis A., Perdikis C. and Takhar H.S. (2004): Effect of thermal radiation on MHD flow. - J. Appl. Math. Comput., vol.153, pp.645-649.

[28]. Ahmed S. and Tridip K.K. (2010): Thermal radiation and magneto hydrodynamic effects on heat and mass transfer chemically reacting fluid with periodic suction. - Int. J. Appl. Math., vol.23, No.5, pp.778-789.

[29]. Prasad V.R., Vasu B., Beg O.A. and Parshad R.D. (2012): Thermal radiation effects on magnetohydrodynamic free convection heat and mass transfer from a sphere in a variable porosity regime. - Commun. Nonlin. Sci. Numer. Simulat., vol.17, pp.654-71.

[30]. Takhar H.S., Chamkha A.J. and Nath G. (2000): Flow and mass transfer on a stretching sheet with a magnetic field and chemically reactive species. - Int. J. Engg. Sci., vol.38, 1303.

[31]. Muthucumarswamy R. and Ganesan P. (2001): First order chemical reaction on flow past an impulsively started vertical plate with uniform heat and mass flux. - Acta Mechanica, vol.147, pp.45-57. 
[32]. Muthucumarswamy R. and Ganesan P. (2002): Effects of suction on heat and mass transfer along a moving vertical surface in the presence of chemical reaction. - Forsch. Ingenieurwes, vol.67, 129.

[33]. Kandasamy R., Periasamy K. and Prabhu K.K.S. (2005): Chemical reaction, heat and mass transfer on MHD flow over a vertical stretching surface with heat source and thermal stratification effects. - Int. J. Heat Mass Transfer, vol.48, No.21-22, pp.4557-4561.

[34]. Ibrahim F.S., Elaiw A.M. and Bakr A.A. (2008): Effect of the chemical reaction and radiation absorption on the unsteady MHD free convection flow past a semi-infinite vertical permeable moving plate with heat source and suction. - Commu. Nonlinear Sci. Numer. Simulat., vol.13, No.6, pp.1056-1066.

[35]. Prakash J., Sivaraj R. and Rushi Kumar B. (2011): Influence of chemical reaction on unsteady MHD mixed convective flow over a moving vertical porous plate. - Int. J. Fluid. Mech., vol.3, pp.1-14.

[36]. Pal D. and Talukdar B. (2011): Combined effects of Joule heating and chemical reaction on unsteady magnetohydrodynamic mixed convection of a viscous dissipating fluid over a vertical plate in porous media with thermal radiation. - Math. Comput. Model., vol.54, pp.3016-3036.

Received: March 29, 2017

Revised: March 15, 2018 